\title{
Periodic forcing of scroll rings and control of Winfree turbulence in excitable media
}

\author{
S. Alonso \\ Abteilung Physikalische Chemie, Fritz-Haber-Institut der Max-Planck-Gesellschaft, Faradayweg 4-6, \\ 14195 Berlin, Germany and Departament de Química Física, Universitat de Barcelona, Av. Diagonal 647, \\ 08028 Barcelona, Spain \\ F. Sagués \\ Departament de Química Física, Universitat de Barcelona, Av. Diagonal 647, 08028 Barcelona, Spain
}

\begin{abstract}
A. S. Mikhailov
Abteilung Physikalische Chemie, Fritz-Haber-Institut der Max-Planck-Gesellschaft, Faradayweg 4-6, 14195 Berlin, Germany
\end{abstract}

(Received 16 November 2005; accepted 19 April 2006; published online 23 June 2006)

\begin{abstract}
By simulations of the Barkley model, action of uniform periodic nonresonant forcing on scroll rings and wave turbulence in three-dimensional excitable media is investigated. Sufficiently strong rapid forcing converts expanding scroll rings into the collapsing ones and suppresses the Winfree turbulence caused by the negative tension of wave filaments. Slow strong forcing has an opposite effect, leading to expansion of scroll rings and induction of the turbulence. These effects are explained in the framework of the phenomenological kinematic theory of scroll waves. (C) 2006 American Institute of Physics. [DOI: 10.1063/1.2203589]
\end{abstract}

Scroll waves are fundamental excitation patterns in three-dimensional (3D) excitable reaction-diffusion media. Discovered by A. T. Winfree in the experiments with the Belousov-Zhabotinsky reaction, ${ }^{1}$ they play an important role in the development of cardiac arrhythmias and heart fibrillation. ${ }^{2,3}$ The Winfree turbulence of scroll waves is due to the instability of their filaments based on the effect of negative tension. It can be suppressed by application of uniform nonresonant forcing with appropriate frequency and amplitude. ${ }^{4}$ Here, a detailed analytical study and numerical simulations on the control of scroll rings and Winfree turbulence by external forcing are presented.

\section{INTRODUCTION}

Excitable media support undamped propagation of pulses, whose profile, amplitude, and speed are all determined by the properties of a medium and do not depend on initial conditions. 5 In two-dimensional (2D) media, such pulses correspond to excitation waves. The fronts of the waves can be curved and spiral waves rotating around small circular core regions are often observed. Scroll waves in 3D media are extensions of spiral waves into the third dimension (Fig. 1). The center of a scroll wave is occupied by a filament, defined by a singularity in phase, and the wave is rotating around it. In planar cross sections normal to the filament, spiral waves are seen. The filament is not necessarily straight; it can be curved and closed into a ring. Moreover, complex wave patterns with filaments forming various knots are also possible. ${ }^{6}$

Instabilities of spiral waves leading to their meandering and break up are known (see Refs. 2 and 5). These instabilities result from the interactions between subsequent propa- gating excitation pulses. They can lead to the development of spiral wave turbulence, recently observed in the experiments with the Belousov-Zhabotinsky reaction. ${ }^{7}$ Similar instabilities can take place for scroll waves in three dimensions. ${ }^{2,8-10}$ However, such 3D patterns may also be subject to a different kind of instability related to the effect of negative tension of filaments. $5,11,12$

If a filament is closed into a ring, it would usually shrink at a rate proportional to its curvature. ${ }^{13}$ Thus, the filament tends to decrease its length and this behavior can be described as an effect of (positive) tension of filaments. Because any curved filament can be locally viewed as consisting of circular fragments, positive tension ensures stability of straight linear filaments.

Already in the early systematic numerical investigations, ${ }^{12}$ it was however found that, depending on the parameters of a medium, scroll rings can also expand at a rate proportional to their curvature. This behavior was also found in the analytical kinematic theory of scroll waves. ${ }^{11}$ This theory predicted that expansion should take place as the excitability of the medium is decreased and the boundary of pulse propagation failure is approached. Under expansion conditions, a filament tends to increase its length and therefore it is characterized by negative line tension. Indeed, any shape perturbation would lead to an increase in the total length of such a filament.

As first noticed in Ref. 11, negative tension of filaments should lead to an instability of a straight filament. Through a process of local stretching and bending of a filament, very complex and potentially chaotic wave patterns were expected to develop. This behavior could not, however, be seen in the early simulations. ${ }^{12}$ The development of the instability of scroll waves under negative filament tension conditions was 


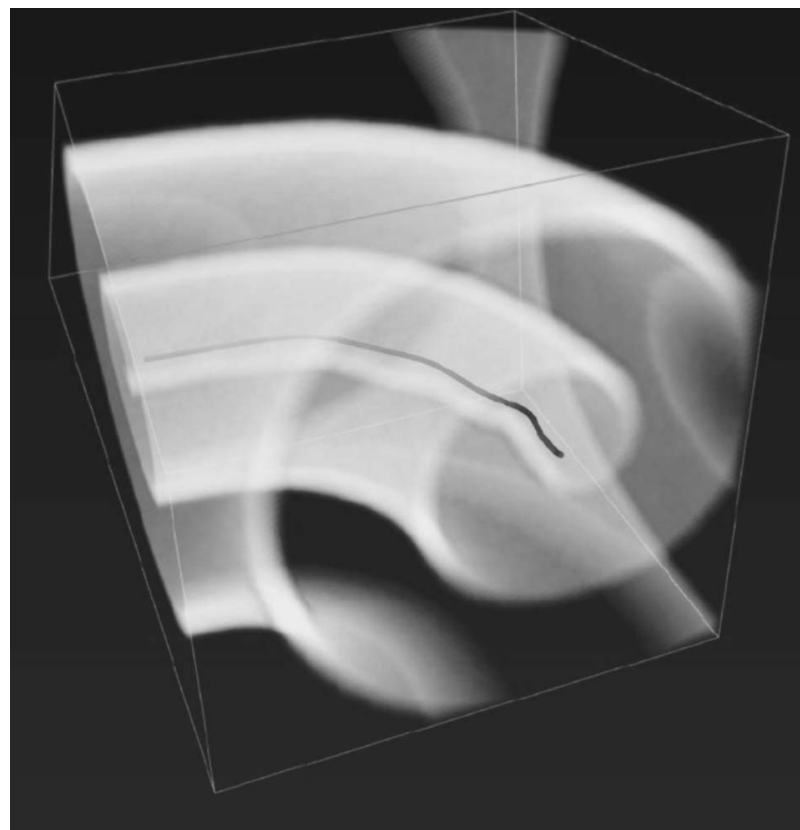

FIG. 1. One-quarter of a scroll ring in a 3D medium, with the filament line shown. This simulation of the Barkley model with the parameters $a=1.1$, $\varepsilon=0.02$, and $b=0.19$ corresponds to the negative tension conditions.

first observed seven years later in numerical simulations by Biktashev $^{14}$ (see also Ref. 15). Subsequently, this kind of complex dynamics was reported by several authors. ${ }^{9,16,17} \mathrm{~A}$ systematic numerical study ${ }^{4,18}$ of the negative tension instability has shown that, in the Barkley model, such instability is found in a broad region in the parameter space and it is therefore robust. Statistical properties of the chaotic dynamics resulting from such an instability, Winfree turbulence of scroll waves, have been discussed ${ }^{18,19}$ and the action of periodic local perturbations on such turbulence has been considered. $^{20}$

Application of weak periodic uniform forcing (through temporal modulation of the medium excitability) provides an easy way to control spiral waves numerically ${ }^{21-23}$ and experimentally. ${ }^{24,25}$ Under resonance conditions, the spiral wave starts to drift along a straight line with the velocity determined by the forcing intensity and the direction determined by the modulation phase. ${ }^{21,26}$ The action of weak periodic resonant forcing on scroll rings was discussed, in the linear approximation in the framework of the kinematic theory, by Abramychev et al. ${ }^{27}$ Action of resonant periodic forcing on collapsing scroll rings was numerically studied by Mantel and Barkley. ${ }^{28}$

In our previous publication, ${ }^{4}$ action of periodic nonresonant forcing on scroll rings and the Winfree turbulence was considered. By numerical simulations of the Barkley model, we have shown that application of rapid forcing (with the frequency higher than the rotation frequency of scroll waves) converts expanding scroll rings into the collapsing ones and suppresses the Winfree turbulence in the medium. On the other hand, application of sufficiently strong slow (subresonant) forcing allows to transform collapsing scroll rings into the expanding ones and induce turbulence in the medium. We have also published, omitting the derivations, some re- sults of a kinematic analysis of this effect, explaining it as a nonlinear renormalization of the filament tension coefficient through periodic forcing. 4

In the next section, a detailed account of our numerical simulations on the action of periodic forcing on scroll rings and turbulence is given. The analytical perturbation theory of scroll rings under the action of weak nonresonant forcing is presented in Sec. III. The comparison of analytical results with the data of numerical simulations is performed in Sec. IV. The article ends with conclusions and a discussion of obtained results.

\section{NUMERICAL INVESTIGATIONS}

Our 3D numerical simulations were performed using the Barkley model ${ }^{29}$

$$
\begin{aligned}
& \frac{\partial u}{\partial t}=D \nabla^{2} u+\frac{1}{\varepsilon} u(1-u)\left(u-\frac{v+b}{a}\right), \\
& \frac{\partial v}{\partial t}=u-v,
\end{aligned}
$$

where variables $u$ and $v$ can be viewed as, respectively, local concentrations of the activator and inhibitor species, positive parameters $a$ and $b$ specify kinetic properties of the system, and the small parameter $\epsilon \ll 1$ represents the ratio of time scales of the (fast) activator and (slow) inhibitor dynamics. Note that the parameter $b$ determines the excitation threshold and thus controls excitability of the medium. We assume here that the inhibitor is not mobile and includes diffusion only into the equation for the activator dynamics. Detailed studies of the Barkley model are available, ${ }^{4,18,30,31}$ identifying regions of subexcitability, excitability, oscillatory, or bistable behaviors in the parameter space and determining various instabilities of the $2 \mathrm{D}$ and $3 \mathrm{D}$ wave structures.

To numerically integrate these equations, they were solved using a finite difference scheme $(\Delta x=0.4)$ for the Laplacian (with the 19 points prescription) and an explicit firstorder Euler method for the time derivative $(\Delta x=0.01)$. Noflux boundary conditions were applied in all simulations. As the initial condition, we usually choose a scroll ring with a circular filament.

The analysis of the simulation data is performed identifying instantaneous shapes and evolution of scroll wave filaments in the medium. To determine locations of a filament, the same procedure as in our previous publications ${ }^{4,18,32}$ has been applied. The boundary of a narrow curved cylinder, representing a filament, is fixed by the conditions $u=0.5$ and $\partial_{t} u=0 .^{9}$

Uniform forcing is introduced into the model by applying weak periodic modulation of the parameter $b$ controlling excitability of the medium,

$$
b(t)=b_{0}+b_{f} \cos \left(\omega_{f} t+\phi_{f}\right),
$$

where $b_{f}$ is the forcing amplitude, $\omega_{f}$ is the forcing frequency, and $\phi_{f}$ is the forcing phase.

Figure 2 shows subsequent snapshots of the evolution of filaments in a numerical simulation. During the initial evolution (panels $\mathrm{A}-\mathrm{E}$ ), the forcing is absent. We see how the 

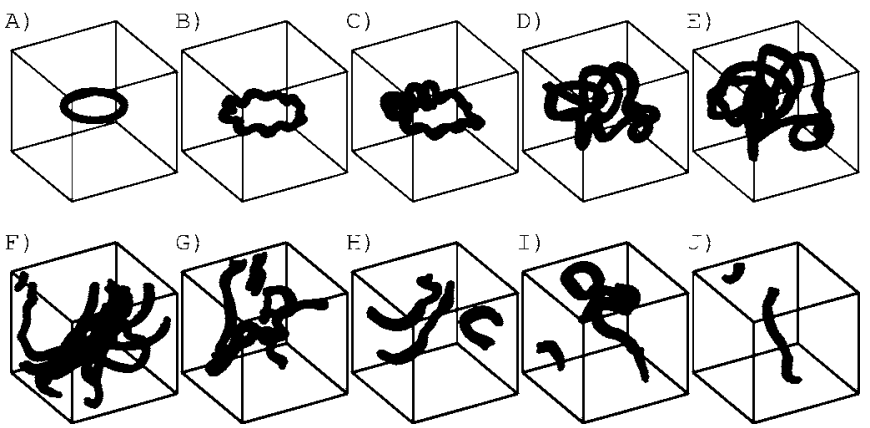

FIG. 2. Snapshots of the development of wave turbulence from an initial scroll ring (A-E), and the elimination of this turbulence by periodic forcing of the excitability $(\mathrm{F}-\mathrm{J})$. The sequence corresponds to the times $t=25,75$, $100,125,150,200,350,500,750$, and 1250. At $t=150$, periodic forcing with $b_{f}=0.015$ and $\omega_{f}=1.1$ is introduced. The waves rotate around the filament with $\omega_{0}=0.99$. Simulation for the medium of size $160 \times 160$ $\times 160$ pixels with $\Delta x=0.4$ and $\Delta t=0.01$. Model parameters are $a=1.0, \varepsilon$ $=0.02$, and $b=0.16$

negative-tension instability of the circular filament develops. Gradually, through elongation and bending, it becomes transformed into a complex tangle filling the volume. It remains single connected, until a boundary is reached and fragmentation begins. Statistical properties of such turbulence have been previously studied by us in Ref. 18. In panel E, rapid forcing is introduced $\left(\omega_{f}>\omega_{0}\right)$. As evidenced by panels F-J in Fig. 2, this leads to the elimination of turbulence from the medium. Small filament fragments shrink and disappear, and the single filament spanning the volume gradually becomes straight.

The principal phenomena underlying elimination of turbulence can be better investigated by considering evolution of a scroll ring in the presence of periodic forcing. To characterize a scroll ring, its mean radius can be used. We define it as

$$
R=\frac{1}{2 \pi} \int_{0}^{2 \pi} d \theta \rho(\theta)
$$

Here $\rho$ and $\theta$ are the local polar coordinates of the filament in its horizontal projection, with the center of the coordinate system coinciding with the center of the scroll ring. Note that for expanding rings, which eventually become transformed into a complex tangle of filaments, this definition is meaningful only at a relatively early stage, as the filament still preserves its circular shape and remains approximately planar.

First, action of rapid forcing on expanding scroll rings is considered. In Fig. 3, the time dependence of the mean radius of a scroll ring is shown for different forcing amplitudes $b_{f}$ and forcing frequencies $\omega_{f}$ (all larger than the rotation frequency $\omega_{0}$ of the scroll). The respective dependence in the absence of forcing is also shown for comparison here, within a time when the shape of the filament is not too complicated to allow the correct definition of the mean radius. We see that, by varying the forcing parameters, it is possible to stabilize expanding rings and to induce their contraction. It can also be seen that, in addition to this principal effect, the forcing leads to periodic modulation of the ring radius, i.e. to breathing of the ring. When the forcing frequency $\omega_{f}$ is de-
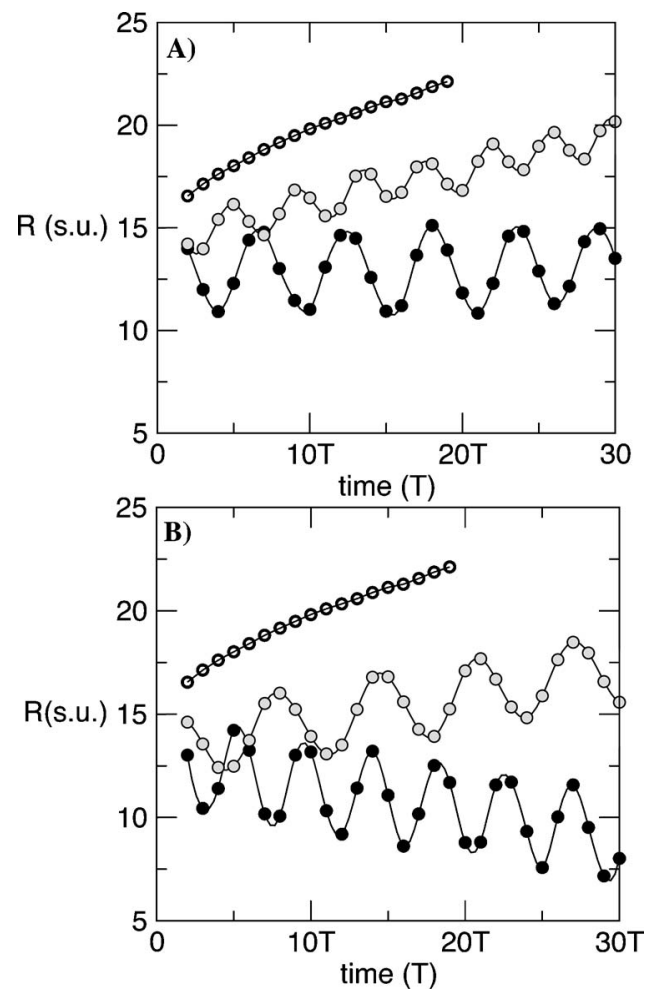

FIG. 3. Time dependence of the mean radius of unforced (empty circles) and forced (black circles) expanding scroll rings. (A) Different forcing frequencies: $\omega_{f}=1.35$ (gray) and $\omega_{f}=1.15$ (black), keeping $b_{f}=0.015$. (B) Different forcing amplitudes: $b_{f}=0.01$ (gray) and $b_{f}=0.02$ (black), keeping $\omega_{f}$ $=1.15$. Each point corresponds to the mean radius averaged over one rotation period $\left(T=6.5, \omega_{0}=0.97\right)$. Simulations for a system of size $160 \times 160$ $\times 160$ pixels with $\Delta x=0.4$ and $\Delta t=0.01$. Model parameters are $a=1.0, \varepsilon$ $=0.02$, and $b=0.17$.

creased, coming closer to $\omega_{0}$, such breathing becomes stronger [Fig. 3(A)]. On the other hand, an increase of the forcing amplitude $b_{f}$ has a strong effect on the expansion rate, but does not lead to a dramatic increase in the amplitude of breathing. In Fig. 4, the evolutions of filament without forcing $\left(\omega_{0}=0.97\right)$ and with a sufficiently strong forcing $\left(\omega_{f}\right.$ $=1.15$ ) are shown. Although turbulence soon develops starting from a scroll ring in the absence of forcing, this does not
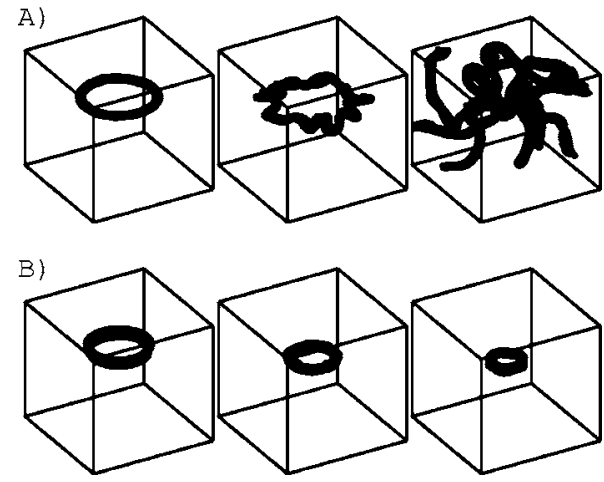

FIG. 4. (A) Snapshots of the filament without forcing for $t$ $=5,15$, and $25 \mathrm{~T}$. (B) Snapshots of the filament under periodic forcing $\left(b_{f}=0.02\right.$ and $\left.\omega_{f}=1.15\right)$ for the same temporal steps. Simulation parameters as in Fig. 3 . 

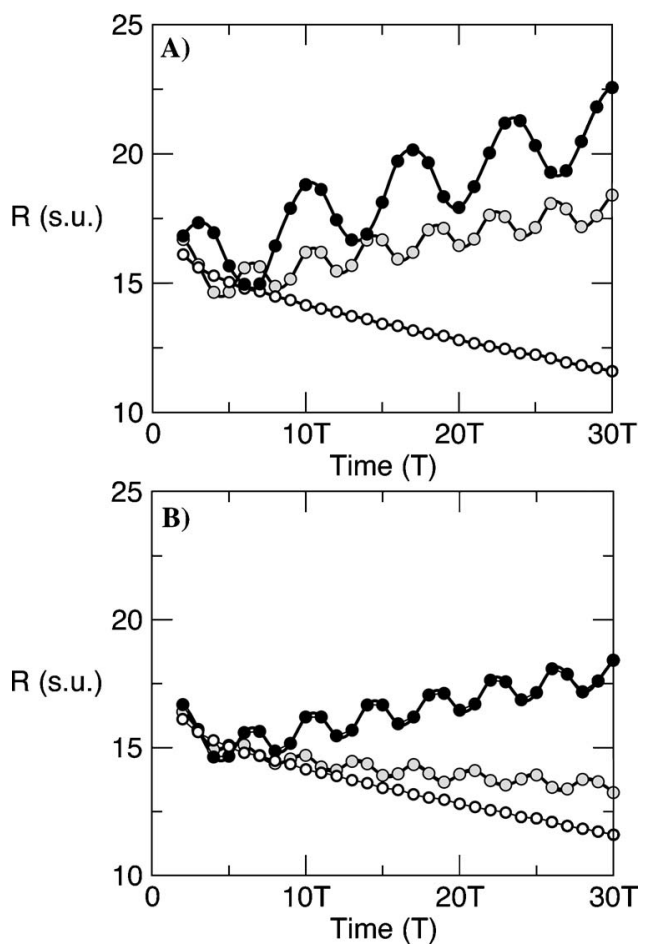

FIG. 5. Time dependence of the mean radius of a unforced (empty circles) and forced (black circles) collapsing scroll rings. (A) Different forcing frequencies $\omega_{f}=0.9$ (gray) and $\omega_{f}=1.0$ (black), keeping $b_{f}=0.01$. (B) Different forcing amplitudes $b_{f}=0.007$ (gray) and $b_{f}=0.01$ (black), keeping $\omega_{f}=1.0$. Each point corresponds to the mean radius averaged over one period of rotation $\left(T=4.5, \omega_{0}=1.40\right)$. Simulations for the medium of size $160 \times 160$ $\times 160$ pixels with $\Delta x=0.4$ and $\Delta t=0.01$. Model parameters are $a=1.0, \varepsilon$ $=0.02$, and $b=0.135$.

happen when forcing is applied. Instead, the ring contracts and eventually disappears.

In Fig. 5, action of slow forcing with $\omega_{f}<\omega_{0}$ on collapsing scroll rings is illustrated. In this case, application of strong enough forcing induces expansion of the rings. As the forced ring expands, it undergoes the same kind of bending instabilities as an expanding ring in the absence of forcing and eventually gives rise to turbulence. The initial stage of this process of slow periodic forcing $\left(\omega_{f}=1.0\right)$ is seen in Fig. 6 , where the respective dependence for a collapsing ring
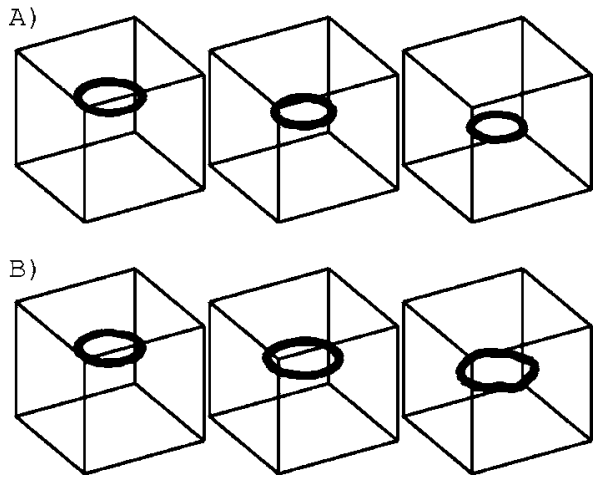

FIG. 6. (A) Snapshots of the filament without forcing for $t$ $=5,15$, and $25 \mathrm{~T}$. (B) Snapshots of the filament under periodic forcing $\left(b_{f}=0.01\right.$ and $\left.\omega_{f}=1.0\right)$ for the same temporal steps. Simulation parameters as in Fig. 5. $\left(\omega_{0}=1.4\right)$ in absence of forcing is additionally shown. Note that slow forcing of collapsing scroll rings also induces their periodic breathing and the amplitude of breathing is increased as the resonance condition $\omega_{f}=\omega_{0}$ is approached. Thus, numerical simulations of the Barkley model with nonresonant forcing suggest that its effect may be understood as consisting of some renormalization of the filament tension in the medium. The filament tension is apparently increased when rapid forcing with $\omega_{f}>\omega_{0}$ is applied and decreased when the forcing is slow $\left(\omega_{f}<\omega_{0}\right)$. The magnitude of the effect increases for stronger forcing amplitudes and when the resonance condition is approached. Additionally, the forcing always induces periodic breathing of the scroll ring, with the amplitude and period of breathing depending on the proximity to the resonance.

In the next section, we construct an approximate analytical theory of forced scroll rings which allows to reproduce these principal numerical observations.

\section{KINEMATICS OF FORCED SCROLL RINGS}

The kinematic theory ${ }^{5,11,21,33}$ provides a phenomenological description of excitation waves in weakly excitable media, when the intervals between subsequent waves are large and the medium undergoes complete recovery before the next excitation pulse arrives. In this approach, the finite width of an excitation wave is not taken into account and the wave is modeled as a (infinitely) thin oriented propagating curve. The motion of this curve is completely determined by its local curvature. If a curve has a free end (the tip), it can grow or contract there, depending on the local curvature at the end point. This simple approach already allows to describe spiral waves and to consider their response to spatial gradients and periodic forcing. ${ }^{21}$ Near the boundary of the subexcitable region, where the rotation period of spiral waves diverges, it yields the power-law scaling with an exponent of $3 / 2$ for the rotation period, in agreement with the recent more detailed analysis. ${ }^{31}$

The kinematic description is also applicable for excitation waves in 3D media. ${ }^{5,11,33}$ In three dimensions, an excitation wave is modeled as an oriented traveling surface which may have an open edge. Again, the motion of the surface and its growth or contraction at the edge are completely determined by the geometry of the surface. Each element of the surface moves in its normal direction with the velocity $V=V_{0}-2 D H$ that depends on the local mean curvature $H$ of the surface (given by an arithmetic average of its two local principal curvatures). At its free edge, the surface grows or contracts at a velocity $G=G_{0}-2 \gamma H_{0}-\gamma^{\prime} \tilde{k}$ that depends not only on the mean surface curvature near the end $\left(H_{0}\right)$, but also on the local geodetic curvature $\widetilde{k}$ of the edge line. Thus, an excitable medium is completely characterized in this kinematic description by a set of five kinematic parameters $V_{0}, G_{0}, D, \gamma$, and $\gamma^{\prime}$. These phenomenological parameters should be found by separate numerical calculations for a particular model of an excitable medium ${ }^{34}$ or determined from the experimental data (as has been done for the spiral waves in the $2 \mathrm{D}$ Belousov-Zhabotinsky medium ${ }^{35}$ ). 


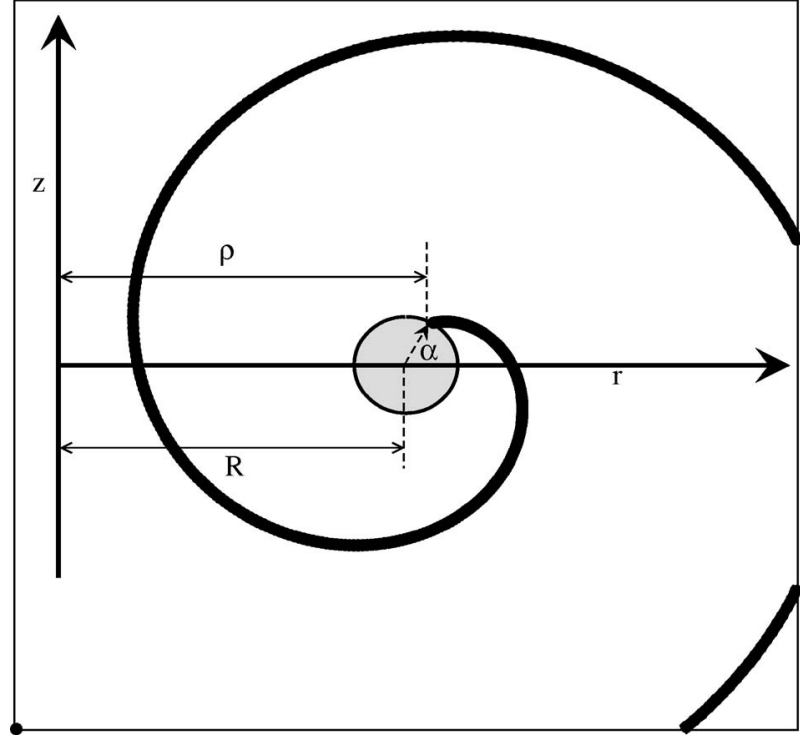

FIG. 7. Meridian cross section of a scroll ring. The gray circle is the wave filament.

Note that the parameter $G_{0}$ plays a special role in this description. Spiral wave solutions in $2 \mathrm{D}$ media and scroll wave solutions in three dimensions exist only if $G_{0}>0$ and their rotation period diverges as $G_{0} \rightarrow 0$. The line $G_{0}=0$ in the parameter space defines the boundary of the subexcitable region and the condition of weak excitability is $G_{0} \ll V_{0}$.

To introduce periodic forcing into the kinematic description, we shall assume that $G_{0}$ is periodically modulated with time,

$$
G_{0}=\bar{G}_{0}\left(1+\epsilon \cos \left(\omega_{f} t+\phi_{f}\right)\right)
$$

where $\omega_{f}$ is the forcing frequency and $\phi_{f}$ is the forcing phase. The modulation is weak and $\epsilon \ll 1$ will be considered as a small parameter of the problem.

In its meridian cross section, a scroll ring represents a spiral whose core corresponds to the filament. The edge line of the scroll is a circle; the geodetic curvature of this line is $\tilde{k}=-\rho^{-1} \cos \alpha$. The mean curvature of the scroll surface at the edge is $H_{0}=\left(k-\rho^{-1} \sin \alpha\right) / 2$, where $k$ is the curvature of the spiral in the cross section, taken at its free end, and $\rho$ is the radius of the edge circle (see Fig. 7). For scroll rings with a large radius $R$, we have $\rho \approx R$.

As shown in previous publications, ${ }^{5,11,33}$ in the quasisteady approximation the dynamics of the scroll ring is governed by a set of four ordinary differential equations for the variables $k, \alpha, \rho$, and $z$, specifying the location of the scroll ring in the direction along its symmetry axis,

$$
\begin{aligned}
\dot{k}= & \zeta\left(\frac{V_{0}}{D}\right)^{1 / 2} k^{3 / 2}\left(G_{0}-\gamma k\right) \\
& +\frac{1}{R}\left(\zeta\left(\frac{V_{0}}{D}\right)^{1 / 2} k^{3 / 2}\left(\gamma \sin \alpha+\left(\gamma^{\prime}-D\right) \cos \alpha\right),\right.
\end{aligned}
$$

$$
\begin{aligned}
\dot{\alpha}= & \zeta\left(V_{0} D\right)^{1 / 2} k^{3 / 2}+k\left(G_{0}-\gamma k\right) \\
& +\frac{1}{R}\left(\gamma \sin \alpha+\left(\gamma^{\prime}-D\right) \cos \alpha\right) k, \\
\dot{\rho}= & -V_{0} \sin \alpha-\frac{1}{R}\left(D+\left(\gamma \sin \alpha+\left(\gamma^{\prime}-D\right) \cos \alpha\right) \cos \alpha\right), \\
\dot{z}= & V_{0} \cos \alpha-\frac{1}{R}\left(\gamma \sin \alpha+\left(\gamma^{\prime}-D\right) \cos \alpha\right) \sin \alpha,
\end{aligned}
$$

where $\zeta=0.685$ is a numerical coefficient.

\section{A. Perturbation analysis}

The evolution equations (5) include two small parameters: the dimensionless forcing amplitude $\epsilon$ and the inverse radius $\kappa=1 / R$ of the scroll ring. In the following, we develop a perturbation theory and construct expansions in terms of these small parameters of the problem.

For convenience, we introduce a formal vector notation $\mathbf{S}=(k, \alpha, \rho, z)$. The components of this vector are the variables used for the characterization of the dynamics of scroll rings. Thus, the four evolution equations are concisely written as

$$
\dot{\mathbf{S}}=\mathbf{f}\left(\mathbf{S}, G_{0}\right)+\kappa \mathbf{h}(\mathbf{S})
$$

with

$$
\begin{gathered}
\mathbf{f}\left(\mathbf{S}, G_{0}\right)=\left(\begin{array}{c}
\zeta\left(\frac{V_{0}}{D}\right)^{1 / 2} k^{3 / 2}\left(G_{0}-\gamma k\right) \\
\zeta\left(V_{0} D\right)^{1 / 2} k^{3 / 2}+k\left(G_{0}-\gamma k\right) \\
-V_{0} \sin \alpha \\
V_{0} \cos \alpha
\end{array}\right), \\
\mathbf{h}(\mathbf{S})=\left(\begin{array}{c}
\Delta_{1} \cos \left(\alpha+\phi_{1}\right) \zeta\left(\frac{V_{0}}{D}\right)^{1 / 2} k^{3 / 2} \\
\Delta_{1} \cos \left(\alpha+\phi_{1}\right) k \\
-D-\Delta_{1} \cos \left(\alpha+\phi_{1}\right) \cos (\alpha) \\
-\Delta_{1} \cos \left(\alpha+\phi_{1}\right) \sin (\alpha)
\end{array}\right),
\end{gathered}
$$

where $\Delta_{1}=\left(\gamma^{2}+\left(\gamma^{\prime}-D\right)^{2}\right)^{1 / 2}$ and $\phi_{1}=\arctan \left(-\gamma /\left(\gamma^{\prime}-D\right)\right)$. Note that $G_{0}=\bar{G}_{0}+\epsilon G_{1}=\bar{G}_{0}\left(1+\epsilon \cos \left(\omega_{f} t+\phi_{f}\right)\right)$.

The vector $\mathbf{S}$ can be expanded into a series in powers of $\epsilon$ and $\kappa$,

$\mathbf{S}=\mathbf{S}_{(0,0)}+\epsilon \mathbf{S}_{(1,0)}+\kappa \mathbf{S}_{(0,1)}+\epsilon \kappa \mathbf{S}_{(1,1)}+\epsilon^{2} \kappa \mathbf{S}_{(2,1)}+\cdots$.

Substituting this expansion into Eq. (6) and equating the terms with the same powers of the small parameters $\epsilon$ and $\kappa$, the following set of dynamical equations is obtained:

$$
\begin{aligned}
& \dot{\mathbf{S}}_{(0,0)}=\mathbf{f}\left(\mathbf{S}_{(0,0)}, \bar{G}_{0}\right), \\
& \dot{\mathbf{S}}_{(0,1)}=\frac{\partial \mathbf{f}\left(\mathbf{S}, G_{0}\right)}{\partial \mathbf{S}} \mathbf{S}_{(0,1)}+\mathbf{h}\left(\mathbf{S}_{(0,0)}\right), \\
& \dot{\mathbf{S}}_{(1,0)}=\frac{\partial \mathbf{f}\left(\mathbf{S}, G_{0}\right)}{\partial \mathbf{S}} \mathbf{S}_{(1,0)}+\frac{\partial \mathbf{f}\left(\mathbf{S}, G_{0}\right)}{\partial G_{0}} G_{1},
\end{aligned}
$$




$$
\begin{aligned}
\dot{\mathbf{S}}_{(1,1)}= & \frac{\partial^{2} \mathbf{f}\left(\mathbf{S}, G_{0}\right)}{\partial \mathbf{S}^{2}} \mathbf{S}_{(1,0)} \mathbf{S}_{(0,1)}+\frac{\partial^{2} \mathbf{f}\left(\mathbf{S}, G_{0}\right)}{\partial \mathbf{S} \partial G_{0}} G_{1} \mathbf{S}_{(0,1)} \\
& +\frac{\partial \mathbf{f}\left(\mathbf{S}, G_{0}\right)}{\partial \mathbf{S}} \mathbf{S}_{(1,1)}+\frac{\partial \mathbf{h}(\mathbf{S})}{\partial \mathbf{S}} \mathbf{S}_{(1,0)}, \\
\dot{\mathbf{S}}_{(2,1)}= & \frac{1}{2} \frac{\partial^{3} \mathbf{f}\left(\mathbf{S}, G_{0}\right)}{\partial \mathbf{S}^{3}} \mathbf{S}_{(1,0)}^{2} \mathbf{S}_{(0,1)}+\frac{\partial^{3} \mathbf{f}\left(\mathbf{S}, G_{0}\right)}{\partial \mathbf{S}^{2} \partial G_{0}} G_{1} \mathbf{S}_{(0,1)} \mathbf{S}_{(1,0)} \\
& +\frac{\partial^{2} \mathbf{f}\left(\mathbf{S}, G_{0}\right)}{\partial \mathbf{S}^{2}} \mathbf{S}_{(1,0)} \mathbf{S}_{(1,1)}+\frac{\partial^{2} \mathbf{f}\left(\mathbf{S}, G_{0}\right)}{\partial \mathbf{S} \partial G_{0}} G_{1} \mathbf{S}_{(1,1)} \\
& +\frac{\partial \mathbf{f}\left(\mathbf{S}, G_{0}\right)}{\partial \mathbf{S}} \mathbf{S}_{(2,1)},
\end{aligned}
$$

where the derivatives are calculated at $\mathbf{S}=\mathbf{S}_{(0,0)}$ and $G_{0}=\bar{G}_{0}$. We have taken into account here that $\mathbf{f}$ is linear on $G_{0}$ and $\mathbf{h}$ does not depend on $G_{0}$.

Next we integrate these equations, starting from the lowest-order terms.

The zeroth-order solution $\mathbf{S}_{(0,0)}$ represents a straight scroll (i.e., the ring with an infinite radius) in absence of forcing. It coincides with a solution for a spiral wave in the 2D medium. For this solution,

$\rho_{(0,0)}=\frac{V_{0}}{\omega_{0}} \cos \left(\omega_{0} t+\phi_{0}\right), \quad z_{(0,0)}=\frac{V_{0}}{\omega_{0}} \sin \left(\omega_{0} t+\phi_{0}\right)$,

where $\phi_{0}$ is the initial phase. The scroll wave rotates with the frequency $\omega_{0}=\zeta\left(V_{0} D\right)^{1 / 2} \bar{G}_{0}^{3 / 2} \gamma^{-3 / 2}$ around a filament of the radius $V_{0} \omega_{0}^{-1}$ :

The first-order term $\mathbf{S}_{(1,0)}$ takes into account in the linear approximation the effect of periodic forcing on straight scroll waves $(\kappa=0)$. Thus, it corresponds to periodic forcing of spiral waves. ${ }^{21}$ Close to the resonance, for $\left|\omega_{f}-\omega_{0}\right| \ll \omega_{0}$, it gives rise to periodic meandering of the spiral wave

$\rho_{(1,0)}=\frac{3}{4} \frac{V_{0} \gamma}{\left(\omega_{f}-\omega_{0}\right)\left(\gamma^{2}+D^{2}\right)^{1 / 2}} \sin \left[\left(\omega_{f}-\omega_{0}\right) t+\phi_{f}-\phi_{0}-\delta\right]$,

$z_{(1,0)}=\frac{3}{4} \frac{V_{0} \gamma}{\left(\omega_{f}-\omega_{0}\right)\left(\gamma^{2}+D^{2}\right)^{1 / 2}} \cos \left[\left(\omega_{f}-\omega_{0}\right) t+\phi_{f}-\phi_{0}-\delta\right]$,

where $\delta=\arctan (-\gamma / D)$. As $\rho=\rho_{(0,0)}+\rho_{(1,0)}$ and $z=z_{(0,0)}$ $+z_{(1,0)}$, the tip of the spiral wave performs a cycloidal motion with two frequencies $\omega_{0}$ and $\omega_{f}-\omega_{0}$.

The other first-order term $\mathbf{S}_{(0,1)}$ takes into account in the linear approximation the effects of curvature $\kappa=1 / R$ of the scroll ring in absence of periodic external forcing. In this order of the perturbation theory, neglecting small terms of order $G_{0} / V_{0}$, we have

$$
\begin{aligned}
& \dot{\rho}_{(0,1)}=-D+\frac{3}{4} \frac{V_{0} \Delta_{1}}{\bar{G}_{0}} \frac{\gamma}{\left(\gamma^{2}+D^{2}\right)^{1 / 2}} \cos \left(\phi_{1}-\delta\right), \\
& \dot{z}_{(0,1)}=-\frac{3}{4} \frac{V_{0} \Delta_{1}}{\bar{G}_{0}} \frac{\gamma}{\left(\gamma^{2}+D^{2}\right)^{1 / 2}} \sin \left(\phi_{1}-\delta\right) .
\end{aligned}
$$

Hence, we reproduce here the old results ${ }^{11}$ for the scroll rings: a scroll ring drifts along its symmetry axis with the velocity $\kappa \dot{z}_{(0,1)}$ and shrinks (or expands, depending on the sign) in the radial direction at the rate $\kappa \dot{\rho}_{(0,1)}$. Substituting explicit expressions for $\Delta_{1}, \phi_{1}$, and $\delta$, we find that the filament tension $\Gamma_{0}$ is determined by the kinematic parameters (see Refs. 5 and 11) as

$$
\Gamma_{0}=D-\frac{3}{4} \frac{V_{0}}{\bar{G}_{0}} \frac{\gamma\left[D\left(\gamma^{\prime}-D\right)+\gamma^{2}\right]}{\gamma^{2}+D^{2}} .
$$

When the tension is positive $\left(\Gamma_{0}>0\right)$, the scroll ring shrinks. If it is negative $\left(\Gamma_{0}<0\right)$, the scroll ring expands.

The second-order contribution $\mathbf{S}_{(1,1)}$ takes into account an interplay between external periodic forcing and the curvature effect of the scroll ring. Keeping only slowly varying terms with the difference frequency $\left(\omega_{f}-\omega_{0}\right)$, we find

$$
\begin{aligned}
k_{(1,1)}= & -\frac{3}{2} \frac{\gamma \Delta_{1}}{\gamma^{2}+D^{2}} \cos \left[\left(\omega_{f}-\omega_{0}\right) t+\phi_{f}-\phi_{0}-\phi_{1}\right] \\
& -\frac{3}{4} \frac{\Delta_{1}}{\left(\gamma^{2}+D^{2}\right)^{1 / 2}} \sin \left[\left(\omega_{f}-\omega_{0}\right) t+\phi_{f}-\phi_{0}-\phi_{1}+\delta\right], \\
\alpha_{(1,1)}= & -\frac{15}{8} \frac{\omega_{0} \gamma^{2} \Delta_{1}}{\bar{G}_{0}\left(\gamma^{2}+D^{2}\right)\left(\omega_{f}-\omega_{0}\right)} \\
& \times \sin \left[\left(\omega_{f}-\omega_{0}\right) t+\phi_{f}-\phi_{0}-\phi_{1}\right] \\
& +\frac{9}{8} \frac{\gamma \omega_{0} \Delta_{1}}{\bar{G}_{0}\left(\gamma^{2}+D^{2}\right)^{1 / 2}\left(\omega_{f}-\omega_{0}\right)} \\
& \times \cos \left[\left(\omega_{f}-\omega_{0}\right) t+\phi_{f}-\phi_{0}-\phi_{1}+\delta\right] .
\end{aligned}
$$

Further, we have

$$
\begin{aligned}
& \dot{\rho}_{(1,1)}=-V_{0} \cos \left(\omega_{0} t\right) \alpha_{(1,1)}+V_{0} \sin \left(\omega_{0} t\right) \alpha_{(1,0)} \alpha_{(0,1)}, \\
& \dot{z}_{(1,1)}=-V_{0} \sin \left(\omega_{0} t\right) \alpha_{(1,1)}-V_{0} \cos \left(\omega_{0} t\right) \alpha_{(1,0)} \alpha_{(0,1)} .
\end{aligned}
$$

Substituting expressions for $\alpha_{(1,1)}, \alpha_{(1,0)}$ and $\alpha_{(0,1)}$, it can be checked that this second-order term gives rise only to oscillations in the radius and vertical position of the ring, induced by external forcing.

Finally, we calculate the third-order coefficients $\mathbf{S}_{(2,1)}$. Thus, we obtain

$$
\begin{aligned}
\dot{k}_{(2,1)}= & -\frac{\gamma}{D} \omega_{0} k_{(2,1)}+\frac{3}{2} \frac{\omega_{0} \gamma}{D} \cos \left(\omega_{f} t+\phi_{f}\right) k_{(1,1)} \\
& -3 \frac{\omega_{0} \gamma^{2}}{\bar{G}_{0} D} k_{(1,0)} k_{(1,1)}+\frac{1}{2} \frac{\omega_{0} \gamma^{2}}{\bar{G}_{0} D} \cos \left(\omega_{f} t+\phi_{f}\right) k_{(1,0)} k_{(0,1)} \\
& -\frac{9}{8} \frac{\omega_{0} \gamma^{3}}{\bar{G}_{0}^{2} D} k_{(1,0)} k_{(1,0)} k_{(0,1)}, \\
\dot{\alpha}_{(2,1)}= & \frac{3}{2} \frac{\omega_{0} \gamma}{\bar{G}_{0}} k_{(2,1)}+\frac{3}{4} \frac{\omega_{0} \gamma^{2}}{\bar{G}_{0}^{2}} k_{(1,0)} k_{(1,1)} \\
& -\frac{3}{16} \frac{\omega_{0} \gamma^{3}}{\bar{G}_{0}^{3}} k_{(1,0)} k_{(1,0)} k_{(0,1)},
\end{aligned}
$$




$$
\begin{aligned}
\dot{\rho}_{(2,1)}= & -V_{0} \cos \left(\omega_{0} t\right) \alpha_{(2,1)}+V_{0} \sin \left(\omega_{0} t\right) \alpha_{(1,0)} \alpha_{(1,1)} \\
& +\frac{1}{2} V_{0} \cos \left(\omega_{0} t\right) \alpha_{(1,0)} \alpha_{(1,0)} \alpha_{(0,1)}, \\
\dot{z}_{(2,1)}= & -V_{0} \sin \left(\omega_{0} t\right) \alpha_{(2,1)}-V_{0} \cos \left(\omega_{0} t\right) \alpha_{(1,0)} \alpha_{(1,1)} \\
& +\frac{1}{2} V_{0} \sin \left(\omega_{0} t\right) \alpha_{(1,0)} \alpha_{(1,0)} \alpha_{(0,1)} .
\end{aligned}
$$

Analyzing the time dependence of the variables yielded by these equations, we can notice that $k_{(2,1)}$ and $\alpha_{(2,1)}$ include only rapidly oscillating with frequencies $\omega_{0}$ and $2 \omega_{f} \pm \omega_{0}$, which are of little interest to us. In the expressions for $\dot{\rho}_{(2,1)}$ and $\dot{z}_{(2,1)}$, the dominant role is played by the second terms because they are proportional to $\left(\omega_{f}-\omega_{0}\right)^{-1}$ and therefore large near the resonance. Retaining only these terms, we find

$$
\begin{aligned}
\dot{\rho}_{(2,1)}= & -\frac{9}{32} \frac{V_{0}}{\bar{G}_{0}} \frac{\gamma^{3} \Delta_{1}}{\left(\gamma^{2}+D^{2}\right)^{3 / 2}} \frac{\omega_{0}}{\omega_{f}-\omega_{0}} \\
& \times\left[\cos \left(\phi_{1}-\delta\right)-\frac{3}{2} \frac{D}{\gamma} \sin \left(\phi_{1}-\delta\right)\right], \\
\dot{z}_{(2,1)}= & \frac{9}{32} \frac{V_{0}}{\bar{G}_{0}} \frac{\gamma^{3} \Delta_{1}}{\left(\gamma^{2}+D^{2}\right)^{3 / 2}} \frac{\omega_{0}}{\omega_{f}-\omega_{0}} \\
& \times\left[\sin \left(\phi_{1}-\delta\right)-\frac{3}{2} \frac{D}{\gamma} \cos \left(\phi_{1}-\delta\right)\right] .
\end{aligned}
$$

\section{B. Summary of analytical results}

The perturbation analysis has allowed us to determine the first terms in the expansion in powers of the forcing amplitude $\epsilon$ and inverse radius $\kappa=1 / R$ of the scroll ring, $\mathbf{S}$ $\approx \mathbf{S}_{(0,0)}+\epsilon \mathbf{S}_{(1,0)}+\kappa \mathbf{S}_{(0,1)}+\epsilon^{2} \kappa \mathbf{S}_{(2,1)}$. We have retained in these terms only the contributions which become dominant near the resonance $\omega_{f}=\omega_{0}$.

To compare with the numerical simulations for the Barkley model, we are primarily interested in the expressions for the rate $\dot{\rho}$ of shrinking (or expansion) of the filament and for the velocity $\dot{z}$ of its vertical motion. Putting together the dominant slowly varying terms with frequency $\left(\omega_{f}-\omega_{0}\right)$ in the perturbation series, we find that $\dot{\rho}$ and $\dot{z}$ are given by

$$
\begin{aligned}
& \dot{\rho}=-\Gamma_{\mathrm{eff}} \frac{1}{R}+\epsilon A \cos \left[\left(\omega_{f}-\omega_{0}\right) t+\phi_{f}-\phi_{0}-\delta\right], \\
& \dot{z}=-\Lambda_{\mathrm{eff}} \frac{1}{R}-\epsilon A \sin \left[\left(\omega_{f}-\omega_{0}\right) t+\phi_{f}-\phi_{0}-\delta\right] .
\end{aligned}
$$

In these equations, $\Gamma_{\text {eff }}$ represents the effective tension coefficient of the filament. It can be written as

$$
\Gamma_{e f f}=\Gamma_{0}+\frac{\epsilon^{2} B}{\omega_{f}-\omega_{0}}
$$

with

$$
\Gamma_{0}=D-\frac{3}{4} \frac{V_{0}}{\bar{G}_{0}} \frac{\gamma\left[D\left(\gamma^{\prime}-D\right)+\gamma^{2}\right]}{\left(\gamma^{2}+D^{2}\right)}
$$

and

$$
B=\frac{9}{32} \frac{V_{0} \gamma^{3} \omega_{0}}{\bar{G}_{0}\left(\gamma^{2}+D^{2}\right)^{2}}\left[\left(D\left(\gamma^{\prime}-D\right)+\gamma^{2}\right)+\frac{3}{2} D\left(2 D-\gamma^{\prime}\right)\right] .
$$

The coefficient $B$ is positive, if $4 D+\gamma^{2} / D>\gamma^{\prime}$. This condition, which we assume in the following, holds for a wide range of kinematic parameters (e.g., when $\gamma^{\prime} \leqslant \gamma$ or $\gamma^{\prime} \leqslant D$ ).

The term $\Gamma_{0}$ yields the filament tension coefficient in absence of forcing, whereas the second term in expression (19) takes into account effective renormalization of the tension coefficient due to periodic external forcing. The renormalization correction is quadratic in the small parameter $\epsilon$ and inversely proportional to $\omega_{f}-\omega_{0}$, which is also a small parameter of the perturbation expansion. To justify the perturbation approximation, the correction must be small, so that $\left(\omega_{f}-\omega_{0}\right) / \omega_{0} \gg \epsilon^{2}$. This renormalization correction becomes significant when the first term $\Gamma_{0}$ is small, i.e., near a boundary in the parameter plane where the contraction of scroll rings is changed into their expansion.

The coefficient $\Lambda_{\text {eff }}$, given by

$$
\begin{aligned}
\Lambda_{\mathrm{eff}}= & \frac{3}{4} \frac{V_{0} \gamma^{2}\left(\gamma^{\prime}-2 D\right)}{\bar{G}_{0}\left(\gamma^{2}+D^{2}\right)}-\frac{9}{32} \frac{\epsilon^{2} \omega_{0}}{\omega_{f}-\omega_{0}} \frac{V_{0} \gamma^{4}}{\bar{G}_{0} D\left(\gamma^{2}+D^{2}\right)^{2}} \\
& \times\left[\left(\gamma^{\prime}-2 D\right)-\frac{3}{2} D\left(1+\frac{D\left(\gamma^{\prime}-D\right)}{\gamma^{2}}\right)\right],
\end{aligned}
$$

determines the velocity and the direction of the drift of a scroll ring along its symmetry axis. Similar to $\Gamma_{\text {eff }}$, it includes renormalization due to periodic forcing, quadratic in the forcing amplitude.

In the limit $R \rightarrow \infty$, the terms with $1 / R$ disappear from Eqs. (17) and (18). The remaining terms are linear in the forcing amplitude $\epsilon$. Here, the coefficient $A$ is

$$
A=\frac{3}{4} \frac{V_{0} \gamma}{\left(\gamma^{2}+D^{2}\right)^{1 / 2}} \text {. }
$$

These terms describe circular oscillations of the filament position in the radial cross section of the ring, i.e., in the plane $(\rho, z)$, which can be characterized as breathing of the scroll ring. Such slow oscillations have frequency $\omega_{f}-\omega_{0}$ and their amplitude (the breathing radius) is given by

$$
R_{1}=\epsilon A /\left|\omega_{f}-\omega_{0}\right| \text {. }
$$

Note that the breathing of the filament corresponds to meandering of spiral waves induced by periodic external forcing. ${ }^{21,23}$ For a large, but finite radius $R$ of the ring, the breathing is superposed with the slow expansion or contraction of the scroll ring filament. In the perturbation theory, relative changes in the radius of the scroll ring filament are assumed to be small. Therefore, the breathing radius must be small as compared with the radius of the ring, $R_{1} \ll R$, which again puts a restriction on the closeness to the resonance condition $\omega_{f}=\omega_{0}$.

The most important results of our analytical investigation is the Eq. (19) for the effective filament tension coefficient $\Gamma_{\text {eff }}$. The periodic forcing of scroll rings leads to a correction in the linear tension coefficient of the filament which is proportional to $\epsilon^{2} /\left(\omega_{f}-\omega_{0}\right)$. When the frequency of 
forcing is larger than the characteristic frequency of rotation of the scroll ring $\left(\omega_{f}>\omega_{0}\right)$, this correction is positive and it increases the tension of the filament. If the filament tension in absence of forcing is negative $\left(\Gamma_{0}<0\right)$ and the scroll ring expands, application of sufficiently strong rapid forcing can make the effective filament tension positive $\left(\Gamma_{\text {eff }}>0\right)$ and thus convert expanding scroll rings into the collapsing ones. On the other hand, if the natural filament tension is positive and the scroll rings contract, application of sufficiently strong slow forcing $\left(\omega_{f}<\omega_{0}\right)$ would allow us change the sign of the filament tension coefficient and make it negative, thus inducing the expansion of scroll rings and, eventually, the Winfree turbulence.

The correction to the filament tension coefficient, due to periodic forcing, is inversely proportional to the distance $\omega_{f}-\omega_{0}$ from the resonance and grows as the resonance condition is approached. It may seem therefore that stabilization of expanding scroll rings and suppression of the Winfree turbulence can be best achieved if one works very close to the resonance condition. This is not, however, true. When the resonance condition $\omega_{f}=\omega_{0}$ is approached, the amplitude of breathing at the difference frequency $\omega_{f}-\omega_{0}$ increases as $1 /\left(\omega_{f}-\omega_{0}\right)$. This large-scale breathing motion is linear in the forcing amplitude $\epsilon$, whereas the renormalization corrections to the filament tension are quadratic in $\epsilon$. Thus, the linear breathing effect is dominating the behavior of scroll rings very close to the resonance.

\section{NUMERICAL TESTS OF KINEMATIC PREDICTIONS}

In this section, we test the main predictions of the kinematic analysis by comparing them with the results of numerical simulations of the Barkley model. It should be noted that the analytical study has been performed previously under a number of assumptions, such as weak excitability (i.e., closeness to the subexcitability boundary), weak forcing, proximity to the resonance, and a large radius of the scroll ring filament. The phenomenological kinematic parameters are not known for the Barkley model. Additionally, forcing in the simulations is never so weak and the scroll radius is never so large, that the conditions of the perturbation theory are fully satisfied. Therefore, direct quantitative comparison between the two descriptions is not possible. Nonetheless, we go beyond merely noticing qualitative agreements between the two classes of results and check whether certain functional laws, independent of particular parameter values, are valid.

According to the kinematic analysis, a periodically forced scroll ring should breath with the frequency $\omega_{f}-\omega_{0}$ where $\omega_{f}$ is the forcing frequency and $\omega_{0}$ is the principal rotation frequency of the scroll wave. Describing numerical simulations in Sec. II, we have noted the presence of periodic breathing. Now, this behavior will be examined in more detail and compared with the respective analytical predictions.

We are not interested here in the developed turbulent regime and consider here only the initial expansion stage of a scroll ring, when it remains approximately circular and the axial symmetry is preserved. Assuming such axial symmetry,

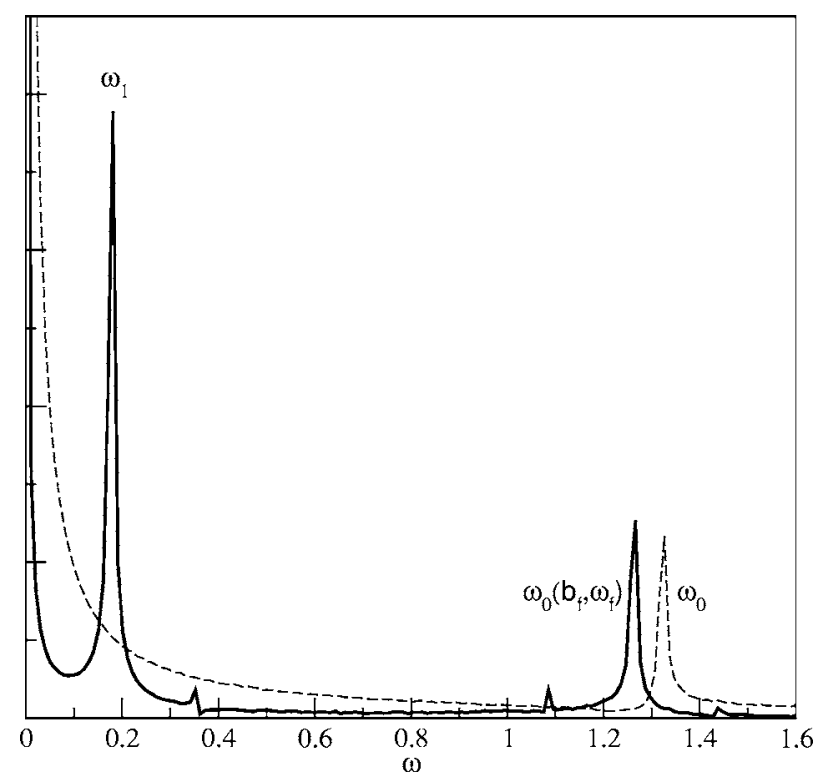

FIG. 8. Power spectra of tip motion for periodically forced (solid line) and unforced (dashed line) scroll rings. The model parameters are $a=1.1, \varepsilon$ $=0.02, b=0.18, b_{f}=0.01$, and $\omega_{f}=1.45$.

the angular variable does not enter into the description of scroll rings and the problem becomes effectively 2D (with the Laplacian written in the cylindrical coordinates):

$$
\begin{aligned}
& \frac{\partial u}{\partial t}=D\left(\frac{\partial^{2} u}{\partial r^{2}}+\frac{1}{r} \frac{\partial u}{\partial r}+\frac{\partial^{2} u}{\partial z^{2}}\right)+\frac{1}{\varepsilon} u(1-u)\left(u-\frac{v+b}{a}\right), \\
& \frac{\partial v}{\partial t}=u-v .
\end{aligned}
$$

The scroll ring is seen in the meridian cross section as a spiral wave, with the cross section of the filament corresponding to the spiral wave core. Further, the free edge of the scroll wave is represented then by a tip of the spiral.

We have performed a series of reduced 2D simulations of forced scroll rings. In each simulation, the tip positions were identified and the tip motion was recorded. By performing fast Fourier transform of this data, the power spectra of the tip motion were computed. An example of such spectrum for the periodically forced expanding scroll ring is shown in Fig. 8. For comparison, we have also displayed here the spectrum or a scroll ring in absence of forcing in the same medium. Without forcing, the spectrum has only one peak at the frequency $\omega_{0}$ representing the rotation frequency of the unforced scroll ring. When forcing is introduced, an additional strong peak at the low frequency $\omega_{1}$ appears. This peak is associated with periodic breathing of a scroll ring. Moreover, we can notice that the peak at a higher frequency, corresponding to the scroll rotation, shifts toward lower frequencies in the presence of forcing. Thus, the rotation frequency of scroll rings depends on the amplitude $b_{f}$ and frequency $\omega_{f}$ of external forcing, $\omega_{0}=\omega_{0}\left(b_{f}, \omega_{f}\right)$. Although the effect is small, this dependence becomes significant close to resonance conditions. Figure 9 shows the actual rotation frequency of scroll rings for different forcing conditions. 


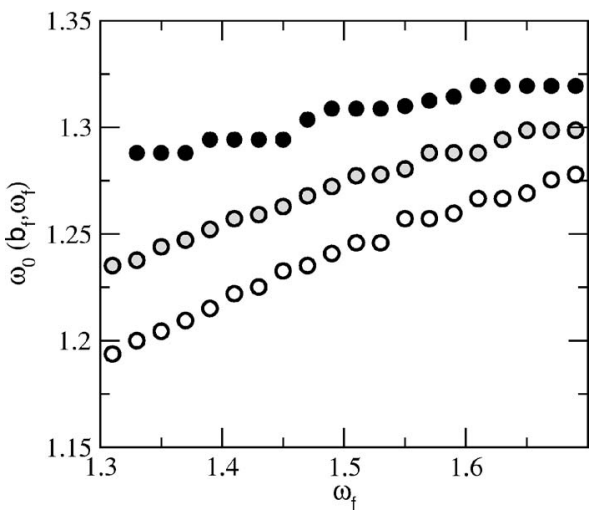

FIG. 9. Primary rotation frequency $\omega_{0}$ of the scroll as function of the forcing frequency $\omega_{f}$ for three different amplitudes of forcing: $b_{f}=0.005$ (black circles), $b_{f}=0.010$ (gray circles), and $b_{f}=0.015$ (white circles). The same model parameters as in Fig. 8 .

Analyzing the spectra, we have identified the low frequency $\omega_{1}$ associated with the filament breathing. The dependences of this frequency on the forcing frequency $\omega_{f}$ for three different amplitudes of forcing are shown in Fig. 10. For comparison, we also display in this figure the analytically predicted dependences $\omega_{1}=\omega_{f}-\omega_{0}\left(b_{f}, \omega_{f}\right)$, where we use the actual values of the principal rotation frequency of the scroll ring shown in Fig. 9. An excellent agreement with the analytical prediction is evident.

The breathing amplitude of forced scroll rings has been further determined. When breathing takes place, in its meridian cross section the filament performs circular motion of some radius $R_{1}$ which can be chosen as the amplitude of breathing. Figure 11 shows the dependence of $R_{1}$ on the forcing frequency for three different amplitudes of forcing. As it is expected from the kinematic result [Eq. (24)], the radius $R_{1}$ should diverge as $R_{1} \propto\left(\omega_{f}-\omega_{0}\right)^{-1}$ when the resonance is approached. To test this prediction, we have plotted in Fig. 11 the curves $R_{1}=C\left(\omega_{f}-\omega_{0}\right)^{-1}$ where the coefficient $C$ was fitted to obtain the same value in the closest point to the resonance. Here, we have used again as $\omega_{0}$ the actual rotation frequencies $\omega_{0}\left(b_{f}, \omega_{f}\right)$ of the forced scroll rings, as displayed in Fig. 9. The agreement is good for weak forcing Fig. 11(A). When the forcing amplitude is increased, the predicted dependence holds only sufficiently closely to the resonance.

A different numerical test of the kinematic theory involves stabilization of expanding scroll rings by periodic forcing. As follows from Eq. (19), expanding scroll rings

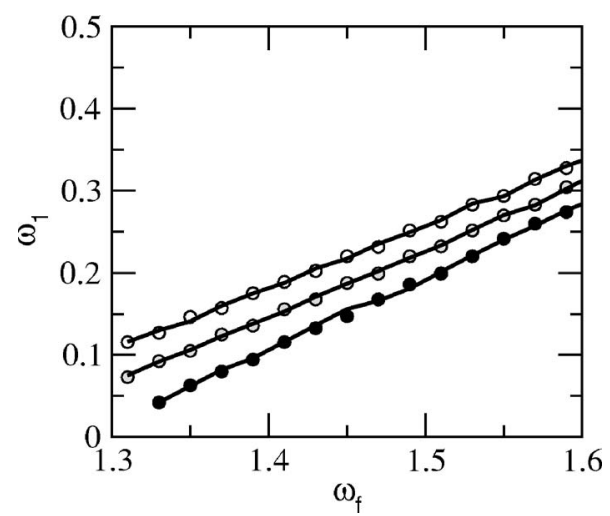

FIG. 10. Breathing frequency $\omega_{1}$ of forced scroll rings as function of the forcing frequency $\omega_{f}$ for three different amplitudes of forcing: $b_{f}=0.005$ (black circles), $b_{f}=0.010$ (gray circles) and $b_{f}=0.015$ (white circles). The curves show the analytical predictions for $\omega_{1}=\omega_{f}-\omega_{0}\left(b_{f}, \omega_{f}\right)$. The same model parameters as in Fig. 8.

$\left(\Gamma_{0}<0\right)$ can be stabilized $\left(\Gamma_{\text {eff }}=0\right)$ by applying forcing (4) with the amplitude proportional to the small parameter $\epsilon$ if its frequency $\omega_{f}$ satisfies the equation

$$
\omega_{f}-\omega_{0}=\frac{B}{\left|\Gamma_{0}\right|} \epsilon^{2} .
$$

In the simulations with the Barkley model, the forcing amplitude is $b_{f}$. Moreover, as we have already noted, the rotation frequency of scroll waves is modified by forcing, $\omega_{0}$ $=\omega_{0}\left(b_{f}, \omega_{f}\right)$. Thus, stable forced scroll rings should be found in the Barkley model along a line in the parameter plane $\left(b_{f}, \omega_{f}\right)$ satisfying the equation

$$
\omega_{f}-\omega_{0}\left(b_{f}, \omega_{f}\right)=F b_{f}^{2},
$$

where $F$ is a numerical coefficient which remains unknown.

To verify this prediction, we have performed simulations of scroll rings (under an assumption of axial symmetry), where periodic forcing of frequency $\omega_{f}$ was applied to expanding scroll rings. By varying the forcing, its critical amplitude $b_{f}$, at which expansion changes to collapse and stable scroll rings are therefore observed, has been determined. Moreover, by analyzing the power spectra of tip motion, the rotation frequency $\omega_{0}\left(b_{f}, \omega_{f}\right)$ of stabilized scroll rings was also computed. In Fig. 12, the difference $\omega_{f}-\omega_{0}\left(b_{f}, \omega_{f}\right)$ is plotted versus the forcing amplitude $b_{f}$ (black circles). The solid curve is the numerical fit of the data to the quadratic
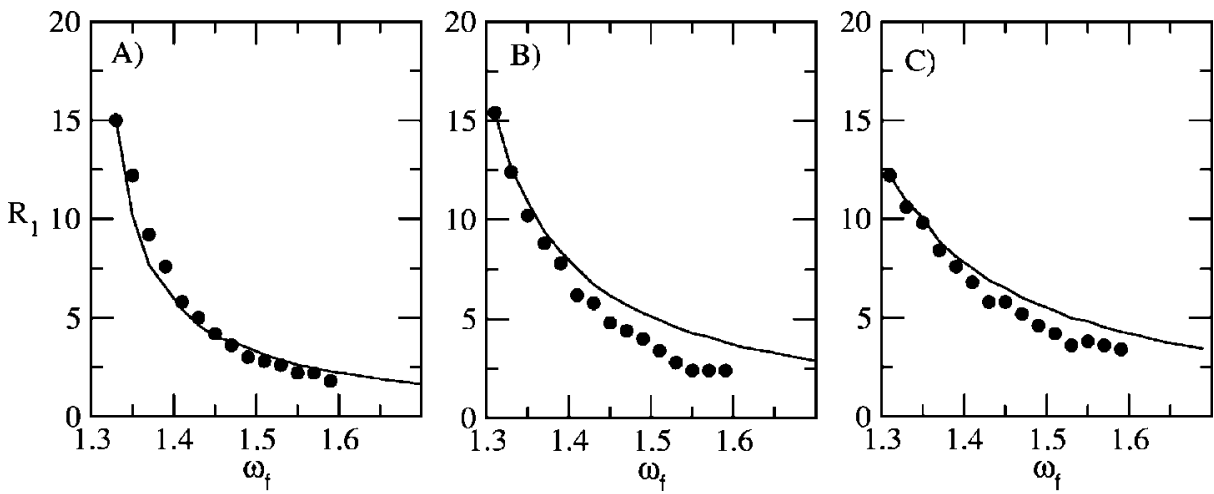

FIG. 11. Breathing radius of scroll rings as function of the forcing frequency $\omega_{f}$, for three different amplitudes of forcing: (A) $b_{f}=0.005$, (B) $b_{f}=0.010$, and (C) $b_{f}=0.015$. The curves show best fits to the analytically predicted dependence $\left(\omega_{f}\right.$ $\left.-\omega_{0}\left(b_{f}, \omega_{f}\right)\right)^{-1}$. 


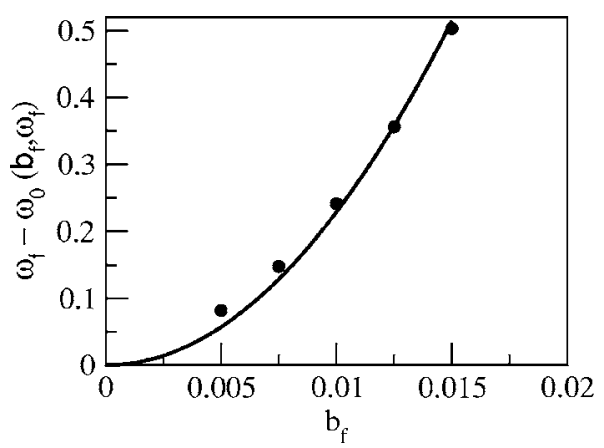

FIG. 12. Numerical test of the kinematic prediction (27). Filled circle symbols indicate expanding scroll rings, stabilized by periodic forcing. The solid curve shows numerical fitting of this data to a quadratic parabola. The same model parameters as in Fig. 8.

parabola with $F=2.3 \times 10^{3}$. We see that the predicted dependence (27) is indeed valid for the Barkley model.

\section{DISCUSSION AND CONCLUSIONS}

The idea of control of scroll rings by periodic forcing has been proposed a long time ago. ${ }^{27}$ However, resonant periodic forcing was considered then as sufficient to realize such control. Scroll rings of a large radius represent spiral waves in the cross section. Under resonant periodic forcing, a spiral wave undergoes a linear drift which direction depends on the forcing phase. Thus, it was believed that, by choosing an appropriate forcing phase, this drift can be made to compensate for the expansion of the scroll ring and thus stabilize it. The simulations with resonant forcing, which we have also performed and which are in the agreement with previous results, ${ }^{28}$ show that stabilization by resonant forcing, proposed in Ref. 27, cannot be reached. First, the rotation frequency of a scroll ring changes under external forcing and it also depends on the ring size, so that it is practically impossible to keep the system under an exact resonance. Moreover, when the negative tension instability is present, the expanding filament deforms and deviates from a circular shape. Depending on the local orientation of various filament fragments, the action of external forcing would then be different and the forcing phase cannot be uniquely adjusted.

The numerical and analytical investigation, which results have been briefly reported in our previous publication ${ }^{4}$ and are described in detail in the present article, is based on the application of nonresonant periodic forcing for the control of scroll rings.

Under the conditions of negative filament tension, scroll rings expand and, through a transverse instability of filaments, give rise to the spatiotemporal regime of Winfree turbulence. By numerical simulations of the Barkley model, we have shown that expansion of filaments can be prevented and Winfree turbulence can be suppressed by applying uniform periodic forcing with a frequency higher than that of the scroll ring and a sufficiently high amplitude. We have also found in our numerical simulations that application of sufficiently strong slow periodic forcing, with the frequency less than the frequency of scroll waves, converts collapsing scroll rings into the expanding ones and thus induces Winfree tur- bulence in the medium. An additional effect of periodic forcing, seen in our simulations, was that the scroll rings breath, i.e., their radius is periodically modulated at a frequency given by the difference of the forcing frequency $\omega_{f}$ and the own rotation frequency $\omega_{0}$ of the scroll ring. In the framework of a phenomenological kinematic approach, the response of scroll rings to periodic forcing has been analytically investigated. The analysis has been performed in a perturbation approximation, using the forcing amplitude and the inverse radius of the scroll ring as small parameters in the perturbation expansions. Moreover, proximity to the resonance condition was assumed in this analysis. The dominant terms, up to the second order in the forcing amplitude and the first order in the inverse radius, have been retained in the perturbation expansion.

We have found that the filament tension coefficient undergoes effective renormalization in the presence of periodic forcing [see Eq. (19)]. The renormalization correction is quadratic in the forcing amplitude. Its sign depends on the relationship between the forcing amplitude $\omega_{f}$ and the rotation frequency $\omega_{0}$ of scroll rings. When $\omega_{f}>\omega_{0}$, the correction is positive and leads to an increase of the filament tension. If $\omega_{f}<\omega_{0}$, it is negative and the filament tension is reduced.

Thus, application of strong enough forcing should convert expanding scroll rings into the collapsing ones. The boundary in the parameter plane, where this transition takes place, is given by Eq. (26). We have shown that there is a good agreement between this analytical prediction and the simulation data for the Barkley model. Further, the kinematic theory predicts breathing of filaments at frequency $\omega_{f}-\omega_{0}$ and with the amplitude inversely proportional to the breathing frequency. This analytical result has been also tested against numerical simulations and good agreement has been found.

Therefore, we conclude that the phenomenological kinematic theory is able to provide an explanation of the phenomena observed in the numerical simulations. Because this theory is not restricted to a particular reaction-diffusion model, its predictions are general. Hence, similar effects of periodic nonresonant forcing are expected for different models of excitable media.

\section{ACKNOWLEDGMENT}

Financial support of the European Union through the Marie Curie Research and Training Network "Universal Principles of Pattern Formation" is gratefully acknowledged.

${ }^{1}$ A. T. Winfree, Science 181, 937 (1973).

${ }^{2}$ F. H. Fenton, E. M. Cherry, H. M. Hastings, and S. J. Evans, Chaos 12, 852 (2002).

${ }^{3}$ A. T. Winfree, Science 266, 1003 (1994).

${ }^{4}$ S. Alonso, F. Sagués, and A. S. Mikhailov, Science 299, 1722 (2003).

${ }^{5}$ A. S. Mikhailov, Foundations of Synergetics I. Distributed Active Systems (Springer, Berlin, 1990).

${ }^{6}$ A. T. Winfree and S. H. Strogatz, Physica D 9, 333 (1983).

${ }^{7}$ L. Q. Zhou and Q. Ouyang, J. Phys. Chem. A 105, 112 (2001).

${ }^{8}$ A. V. Panfilov and P. Hogeweg, Phys. Rev. E 53, 1740 (1996).

${ }^{9}$ F. H. Fenton and A. Karma, Phys. Rev. Lett. 81, 481 (1998).

${ }^{10}$ Z. L. Qu, F. G. Xie, and A. Garfinkel, Phys. Rev. Lett. 83, 2668 (1999).

${ }^{11}$ P. K. Braznhik, V. A. Davydov, V. S. Zykov, and A. S. Mikhailov, Sov. Phys. JETP 66, 984 (1987).

${ }^{12}$ A. V. Panfilov and A. N. Rudenko, Physica D 28, 215 (1987). 
${ }^{13}$ J. P. Keener and J. J. Tyson, Science 239, 1284 (1988).

${ }^{14}$ V. N. Biktashev, A. V. Holden, and H. Zhang, Philos. Trans. R. Soc. London, Ser. A 347, 611 (1994).

${ }^{15}$ V. N. Biktashev, Int. J. Bifurcation Chaos Appl. Sci. Eng. 8, 677 (1998).

${ }^{16}$ R. A. Gray and J. Jalife, Chaos 8, 65 (1998).

${ }^{17}$ F. Chavez, R. Kapral, G. Rosseau, and L. Glass, Chaos 11, 757 (2001).

${ }^{18}$ S. Alonso, R. Kähler, A. S. Mikhailov, and F. Sagués, Phys. Rev. E 70, 056201 (2004).

${ }^{19}$ R. M. Zaritski, S. F. Mironov, and A. M. Pertsov, Phys. Rev. Lett. 92, 168302 (2004).

${ }^{20}$ H. Zhang, Z. Cao, N. Wu, H. Ying, and G. Hu, Phys. Rev. Lett. 94, 188301 (2005).

${ }^{21}$ A. S. Mikhailov, V. A. Davydov, and V. S. Zykov, Physica D 70, 1 (1994).

${ }^{22}$ A. Schrader, M. Braune, and H. Engel, Phys. Rev. E 52, 98 (1995).

${ }^{23}$ R. M. Mantel and D. Barkley, Phys. Rev. E 54, 4791 (1996).

${ }^{24}$ O. Steinbock, V. Zykov, and S. C. Müller, Nature (London) 366, 322 (1993).
${ }^{25}$ M. Braune, A. Schrader, and H. Engel, Chem. Phys. Lett. 222, 358 (1994).

${ }^{26}$ K. I. Agladze, V. A. Davydov, and A. S. Mikhailov, JETP Lett. 45, 767 (1987)

${ }^{27}$ A. Yu. Abramychev, V. A. Davydov, and A. S. Mikhailov, Biophysics (USSR) 35, 520 (1990).

${ }^{28}$ R. M. Mantel and D. Barkley, Physica D 149, 107 (2001).

${ }^{29}$ D. Barkley, M. Kenss, and L. S. Tuckerman, Phys. Rev. A 42, 2489 (1990).

${ }^{30}$ D. Barkley, Phys. Rev. Lett. 72, 164 (1994).

${ }^{31}$ H. Henry and V. Hakim, Phys. Rev. Lett. 85, 5328 (2000).

${ }^{32}$ S. Alonso, J. M. Sancho, and F. Sagués, Phys. Rev. E 70, 067201 (2004).

${ }^{33}$ A. S. Mikhailov, Chaos, Solitons Fractals 5, 673 (1995).

${ }^{34}$ A. S. Mikhailov and V. S. Zykov, Physica D 52, 379 (1991).

${ }^{35}$ O. U. Kheowan, V. Gaspar, V. S. Zykov, and S. C. Muller, Phys. Chem. Chem. Phys. 3, 4747 (2001). 